\title{
Benefits of COMPLETE revascularization
}

$c 4$

it appears
appropriate
to recommend
complete
revasculari-
zation for
patients
similar to those
included in the
COMPLETE

trial

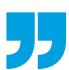

In patients with ST-segment elevation myocardial infarction (STEMI) and multivessel coronary artery disease, a strategy of revascularizing both the culprit lesion and any angiographically significant nonculprit lesions achieves better outcomes than a strategy of revascularizing only the culprit lesion. This finding comes from the COMPLETE trial and was presented at the ESC Congress 2019 in Paris, France.

Patients who require percutaneous coronary intervention (PCI) for STEMI often have multivessel disease with angiographically significant lesions in addition to the lesion that caused the acute event. The aim of the COMPLETE trial was to determine whether these nonculprit lesions should be routinely revascularized or managed conservatively by medical therapy.

A total of 4,041 patients from 140 centres in 31 countries were enrolled. These patients, who had STEMI and multivessel coronary artery disease and had undergone successful culprit-lesion PCI, were randomly assigned to a strategy of complete revascularization involving

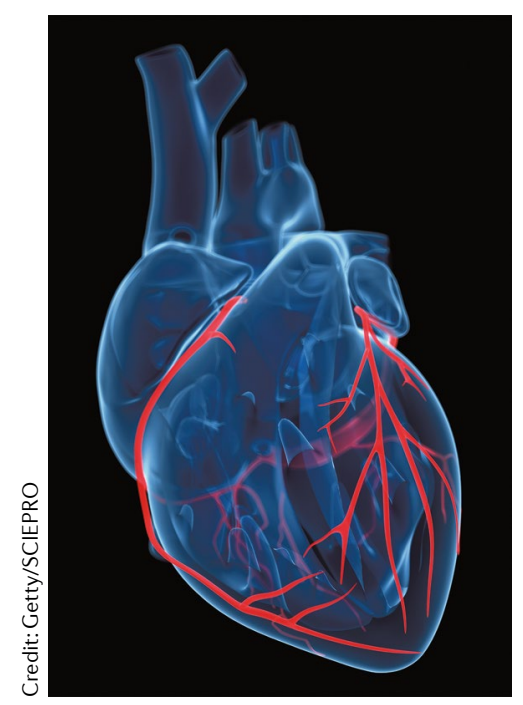

PCI of angiographically significant nonculprit lesions or no further revascularization. Nonculprit lesions were deemed angiographically significant if they were associated with $\geq 70 \%$ stenosis of the vessel diameter or with $50-69 \%$ stenosis accompanied by a fraction flow reserve measurement of $\leq 0.80$.

After nonculprit-lesion PCI, $90.1 \%$ of the patients in the completerevascularization group had a SYNTAX score of 0 , indicating no angiographically significant disease remaining. The crossover rate between the two treatment groups was low $(<5 \%)$.

At follow-up (median 3 years), the first co-primary end point (a composite of cardiovascular death or myocardial infarction) had occurred in $7.8 \%$ of the completerevascularization group and $10.5 \%$ of the culprit-lesion-only PCI group (HR 0.74, 95\% CI 0.60-0.91, $P=0.004)$. This result was driven by a significantly lower incidence of new myocardial infarction in the complete-revascularization group than in the culprit-lesion-only PCI group (5.4\% versus $7.9 \%$; HR 0.68 , $95 \%$ CI $0.53-0.86)$. The incidence of death from cardiovascular causes was not significantly different between the two groups (2.9\% versus $3.2 \%$ ).

The second co-primary outcome (a composite of cardiovascular death, myocardial infarction or ischaemia-driven revascularization) occurred in $8.9 \%$ of the completerevascularization group and $16.7 \%$ of the culprit-lesion-only PCI group (HR 0.51, 95\% CI 0.43-0.61, $P<0.001)$.

The risk of adverse events (including major bleeding, stroke and stent thrombosis) was similar in the two groups, supporting the safety of an additional procedure in the complete-revascularization group. Contrast-associated acute kidney injury occurred in 30 patients in the complete-revascularization group and in 19 patients in the culprit-lesion-only PCI group (OR 1.59, 95\% CI 0.89-2.84, $P=0.11$ ).

Of note, for both co-primary outcomes, the benefit of complete revascularization was consistent whether the nonculprit-lesion PCI was intended to be undertaken during the index hospitalization or subsequent to hospital discharge.

The investigators calculate that, over a period of 3 years, the number needed to treat to prevent one cardiovascular death or myocardial infarction is 37 patients, and the number needed to treat to prevent one cardiovascular death, myocardial infarction or ischaemia-driven revascularization is 13 patients.

"Since this strategy appears to be safe and reduces the risk of the composite outcome of cardiovascular death or recurrent myocardial infarction, as well as the risk of future revascularization, it appears appropriate to recommend complete revascularization for patients similar to those included in the COMPLETE trial," comment Lars Køber and Thomas Engstrøm in an editorial that accompanied the trial publication in The New England Journal of Medicine. However, "patients participating in trials are different from sicker patients seen in the clinical setting, and extrapolation of the results to patients with a greater risk of complications may not be safe," they caution.

Gregory B. Lim ORIGINAL ARTICLE Mehta, S. R. et al. Complete revascularization with multivessel PCI for myocardial infarction. N. Engl.J.Med. https://doi.org/10.1056/ NEJMoa1907775 (2019) RELATED ARTICLE Vogel, B. et al. Reperfusion strategies in acute myocardial infarction and multivessel disease. Nat. Rev. Cardiol. 14, 665-678 (2017) 